\title{
Emittance growth and halo formation in the relaxation of mismatched beams
}

\author{
Tarcisio N. Teles, ${ }^{*}$ Renato Pakter, ${ }^{\dagger}$ and Yan Levin ${ }^{\ddagger}$ \\ Instituto de Física, Universidade Federal do Rio Grande do Sul, Caixa Postal 15051, 91501-970, Porto Alegre, RS, Brazil
}

(Received 15 June 2010; published 11 November 2010)

\begin{abstract}
In this paper, a simplified theoretical model that allows prediction of the final stationary state attained by an initially mismatched beam is presented. The proposed stationary state has a core-halo distribution. Based on the incompressibility of the Vlasov phase-space dynamics, the core behaves as a completely degenerate Fermi gas, where the particles occupy the lowest possible energy states accessible to them. On the other hand, the halo is given by a tenuous uniform distribution that extends up to a maximum energy determined by the core-particle resonance. This leads to a self-consistent model in which the beam density and self-fields can be determined analytically. The theory allows one to estimate the emittance growth and the fraction of particles that evaporate to the halo in the relaxation process. Self-consistent $N$-particle simulation results are also presented and are used to verify the theory.
\end{abstract}

DOI: 10.1103/PhysRevSTAB.13.114202

PACS numbers: 41.85.Ja, 41.75.-i, 05.70.Ln

\section{INTRODUCTION}

In experiments that require the transport of intense beams, space-charge forces make it virtually impossible to launch a beam with a distribution that corresponds to an exact equilibrium state. As a consequence, as the particles are transported the beam will tend to relax towards a stationary state [1,2]. Along this process, effects such as emittance growth and halo formation are expected to occur. These effects are very detrimental because they limit beam efficiency and may be responsible for particle losses which can cause wall damage and activation. Therefore, a quantification of the amount of emittance growth and halo formation that can be expected becomes an important issue in the design of such systems. In order to estimate these, a good knowledge of the mechanisms that lead to beam relaxation and, especially, of the final stationary state reached by the beam is necessary.

In general, injected beams may deviate from the equilibrium state because of various effects, such as envelope mismatches [3-9], off-axis motion [8,10-13], nonuniformities in the beam distribution [14-20], and forces due to the surrounding conductors [21-23]. Among all these effects, the one that has attracted most of the attention is the envelope mismatch because it is believed to be a major cause of emittance growth and halo formation. For mismatched beams, an unbalance between the focusing force due to the external applied field and the defocusing forces due to space charge and thermal effects, causes the whole beam to oscillate in a coherent breathing mode. Some single beam particle trajectories resonate with this mode, gaining a lot of energy to form the halo. Based on a low dimensional particle-core model it is possible to observe

\footnotetext{
*teles@if.ufrgs.br

pakter@if.ufrgs.br

*levin@if.ufrgs.br
}

this resonance process and to determine the maximum range of halo particles [3-5]. Because of conservation of energy, as the halo is being formed the particles that remain in the core lose energy and the amplitude of the breathing mode decreases. Eventually, halo formation ceases and the stationary state is reached. The whole scenario is analogous to an evaporative cooling process where the core particles cool down via evaporation of hot, energetic halo particles. The thermodynamic equilibrium that corresponds to the Maxwell-Boltzmann distribution $[24,25]$ is not expected to be attained in this process because the beam dynamics is collisionless [26-29]. In fact, in the particular case of an initially mismatched high-intensity cold beam, it has been shown that the final stationary state can be very well modeled by a completely cold dense core surrounded by a cloud of energetic particles that carry all the beam emittance [8,9]. From this model, one can successfully determine the total emittance growth and the fraction of particles that form the halo in the stationary state.

In the case of beams with a finite initial emittance, however, the assumption of a completely cold core for the relaxed state is no longer correct. The existence of emittance in the initial distribution indicates that the beam occupies a finite volume in the phase space. Because the Vlasov dynamics that governs beam evolution is incompressible, this volume has to be preserved. Hence, the occupation of low-energy regions of the phase space by the particles as the core progressively cools down is limited by the finite density of the initial distribution in phase space, which is not compatible with a completely cold core. In other words, although we are dealing with purely classical particles, the conservation of volume in the phase space imposed by the Vlasov equation leads to a Pauli-like exclusion principle for the beam particles. Taking this into account, in this paper we propose that the stationary state for the core corresponds to a completely degenerate Fermi gas, where the particles occupy the lowest possible energy 
states accessible to them. This leads to a self-contained model where the beam density and self-fields can be determined analytically as a function of two parameters - the core size and the halo density. These parameters are, in turn, readily obtained by numerically solving two algebraic equations that correspond to the conservation of the total number of particles and the energy of the system. The results are compared with self-consistent $\mathrm{N}$-particle simulations and a good agreement is found for the density of the stationary state and the emittance growth. In the simulations, the emittance growth is shown to be weakly dependent on the details of the initial beam distribution. The model is also used to estimate the fraction of particles that will evaporate to form the halo. It is worth noting that a more detailed analysis shows that the core distribution is indeed not fully degenerate, but more closely represented by a series of low temperature Fermi-Dirac distributions $[26,30]$. If on one hand such a representation is capable of describing the stationary state in great detail, on the other hand it demands more involved computation and requires an equally detailed knowledge of the initial distribution. In this regard, the model proposed here is a simplification which, however, provides a fair description of the stationary state and that is only based on the knowledge of rms quantities of the initial distribution.

The paper is organized as follows. In Sec. II we present the model for the space-charge beam transport and its fundamental equations. In Sec. III, we introduce the proposed final stationary distribution and analytically derive its density and self-field functions. Based on the conservation of particles and energy in the beam transport, we find two algebraic equations that have to be solved in order to determine the stationary state without any adjustable parameter. The expressions for the emittance growth and the halo fraction are obtained from the theory. In Sec. IV, we present numerical results and test the theory against the self-consistent $N$-particle simulations. Finally, in Sec. V, we conclude the paper.

\section{BEAM MODEL AND EQUATIONS}

We consider an unbunched beam propagating with a constant axial velocity $\beta_{b} c$ along the inner channel of a circular grounded conducting pipe of radius $r_{w}$; the beam is focused by a uniform solenoidal magnetic field of magnitude $B_{z}$. Both the pipe and the focusing field are aligned with the $z$ axis. Given the uniform motion along $z$, we define a longitudinal coordinate $s=\beta_{b} c t$ that plays the role of time in the system. It is convenient to work in the Larmor frame of Ref. [31], which rotates with respect to the laboratory frame with the angular velocity $\Omega_{L}=$ $q B_{o} / 2 \gamma_{b} m c$, where $q, m$, and $\gamma_{b}=\left(1-\beta_{b}^{2}\right)^{-1 / 2}$ are, respectively, the charge, mass, and relativistic factor of the beam particles. In the paraxial approximation, the beam distribution function $f(\mathbf{r}, \mathbf{v}, s)$ evolves according to the Vlasov-Maxwell system [31],

$$
\begin{gathered}
\frac{\partial f}{\partial s}+\mathbf{v} \cdot \nabla f+\left(-k_{0}^{2} \mathbf{r}-\nabla \psi\right) \cdot \nabla_{\mathbf{v}} f=0, \\
\nabla^{2} \psi=-\frac{2 \pi K}{N} n(\mathbf{r}, s),
\end{gathered}
$$

where $n(\mathbf{r}, s)=\int f d \mathbf{v}$ is the beam density profile, $k_{0}=$ $q B_{z} / 2 \gamma_{b} \beta_{b} m c^{2}$ is the vacuum phase advance per unit axial length which determines the focusing field strength, $K=$ $2 q^{2} N / \gamma_{b}^{3} \beta_{b}^{2} m c^{2}$ is the beam perveance that is a measure of the beam intensity, $N=\int f d \mathbf{r} d \mathbf{v}=$ const is the conserved number of particles per unit axial length, $\mathbf{r}$ is the position vector in the transverse plane, and $\mathbf{v} \equiv d \mathbf{r} / d s$. As discussed in the Introduction, it is exactly because the beam evolves according to the Vlasov equation (1), that the total phase-space volume occupied by the particles has to be conserved. In Eqs. (1) and (2), $\psi$ is a normalized potential that incorporates both self-electric and self-magnetic field interactions. Because of the presence of the pipe surrounding the beam, the self-field potential satisfies the boundary condition $\psi\left(r=r_{w}\right)=0$. In view of the axisymmetry of the external focusing field and taking into consideration only axisymmetric (breathing) envelope modes, we assume that the beam distribution has no $\theta$ dependence, so that $f=$ $f\left(r, v_{r} ; v_{\theta} ; s\right)$, where the angular velocity $v_{\theta}$ is a constant of motion for the beam particles. For the Vlasov dynamics, if the distribution function only depends on the phasespace variables through the single particle energy, i.e., $f(\mathbf{r}, \mathbf{v})=f(\varepsilon)$, where

$$
\varepsilon(\mathbf{r}, \mathbf{v})=\frac{v^{2}}{2}+\frac{k_{0}^{2} r^{2}}{2}+\psi(r)
$$

it will be stationary. If it is not stationary, the distribution will vary as a function of $s$, tending to relax to a stationary state.

The beam envelope $r_{b}=\left[2\left\langle r^{2}\right\rangle\right]^{1 / 2}$ is a measure of the transverse size of the beam and evolve according to [31]

$$
r_{b}^{\prime \prime}+k_{0}^{2} r_{b}-\frac{K}{r_{b}}-\frac{\epsilon^{2}}{r_{b}^{3}}=0,
$$

where the emittance of the beam is defined as

$$
\epsilon=2\left[\left\langle r^{2}\right\rangle\left\langle v^{2}\right\rangle-\left\langle r v_{r}\right\rangle^{2}\right]^{1 / 2},
$$

the prime denotes derivative with respect to $s$, the angled brackets represent the average over the beam distribution, and $v=\left(v_{r}^{2}+v_{\theta}^{2}\right)^{1 / 2}$. While for equilibrium beam distributions the emittance is a conserved quantity, for a nonstationary beam the emittance $\epsilon=\epsilon(s)$ generally grows as the beam relaxes towards the stationary state (although the emittance can decrease as in the case of an initially matched semi-Gaussian beam as in Ref. [24]). It is clear from Eq. (4) that there is a competition between the focusing force imposed by the external magnetic field and the defocusing forces due to space charge and emittance. For matched beams these forces are balanced in such 
a way that the beam envelope remains mostly constant along the transport. Equating $r_{b}^{\prime \prime}=0$ in Eq. (4) we obtain the matched beam envelope,

$$
r_{b}^{*}=\left[\frac{K+\left(K^{2}+4 k_{0}^{2} \epsilon^{2}\right)^{1 / 2}}{2 k_{0}^{2}}\right]^{1 / 2} .
$$

More generally, however, the initial distribution will have a mismatched envelope. In this case, the envelope will start to oscillate due to the unbalanced focusing and defocusing forces, and will start to induce halo formation as described by the particle-core model [3-5]. In order to quantify the initial beam envelope mismatch, we define a mismatch parameter as given by $\mu \equiv r_{b}(0) / r_{b}^{*}(0)$.

A quantity that plays a key role in the determination of the final stationary state of the beam is its average energy per particle. This is given by

$$
\mathcal{E}=\frac{\left\langle v^{2}\right\rangle}{2}+\frac{k_{0}^{2}\left\langle r^{2}\right\rangle}{2}+\mathcal{E}_{\psi},
$$

and is conserved along the transport. In Eq. (7), $\mathcal{E}_{\psi}$ is the beam self-field energy per particle given by [31]

$$
\mathcal{E}_{\psi}=\frac{1}{4 \pi K} \int|\nabla \psi|^{2} d \mathbf{r}=\frac{1}{2 K} \int_{0}^{r_{w}}\left(\frac{\partial \psi}{\partial r}\right)^{2} r d r .
$$

The aim in the next sections is to determine the final stationary state achieved by a beam of known initial distribution.

\section{DETERMINING THE FINAL STATIONARY STATE}

\section{A. Initial beam with a waterbag distribution}

We start our analysis by considering a beam whose initial distribution corresponds to a phase-space waterbag. That is, the particles are uniformly distributed up to a maximum radius $r_{m}$ and a maximum speed $v_{m}$ [32],

$$
f_{0}(\mathbf{r}, \mathbf{v})=\frac{N}{\pi^{2} \epsilon_{0}^{2}} \Theta\left(r_{m}-r\right) \Theta\left(v_{m}-v\right),
$$

where $r$ is the radial coordinate in the transverse direction, $v=\left(v_{r}^{2}+v_{\theta}^{2}\right)^{1 / 2}$ is the magnitude of the velocity in the transverse direction, $\Theta(x)$ is the Heaviside step function, and $\epsilon_{0}=\epsilon(0)=r_{m} v_{m}$ is the initial beam emittance. This distribution is represented in Fig. 1(a). Its energy per particle can be readily computed by solving the Poisson equation and using Eqs. (7) and (8) to give

$$
\mathcal{E}_{0}=\frac{v_{m}^{2}}{4}+\frac{k_{0}^{2} r_{m}^{2}}{4}+\frac{K}{8}-\frac{K}{2} \log \left(\frac{r_{m}}{r_{w}}\right)
$$

The waterbag distribution given by Eq. (9) is quite convenient for our discussion because it has the property that all the occupied regions in phase space have the same density $N / \pi^{2} \epsilon_{0}^{2}$. Hence, as the beam relaxes, the incompressibility of the Vlasov dynamics will limit the occupation of the lower energy states available to the progressively colder core to this density value. In the final stationary state, therefore, the core will resemble a degenerate Fermi gas of density $N / \pi^{2} \epsilon_{0}^{2}$ that extends up to a Fermi energy $\varepsilon_{F}$ in the phase space. Such a core distribution is equivalent to a waterbag distribution in energy [2,31] that extends up to $\varepsilon_{F}$ and has the prescribed occupation density $N / \pi^{2} \epsilon_{0}^{2}$. The value of $\varepsilon_{F}$ is yet unknown, but will be determined self-consistently. As for the halo, the particle-core model allows us to determine the maximum radius that the halo particles can attain, $r_{h}$ [3,5]. Since the particle located at $r_{h}$ represents the outermost one, we can easily determine its energy as $\varepsilon_{h}=k_{0}^{2} r_{h}^{2} / 2-$ $K \log \left(r_{h} / r_{w}\right)$. While for initially cold beams it was found that the halo particles tend to stay along the separatrix of
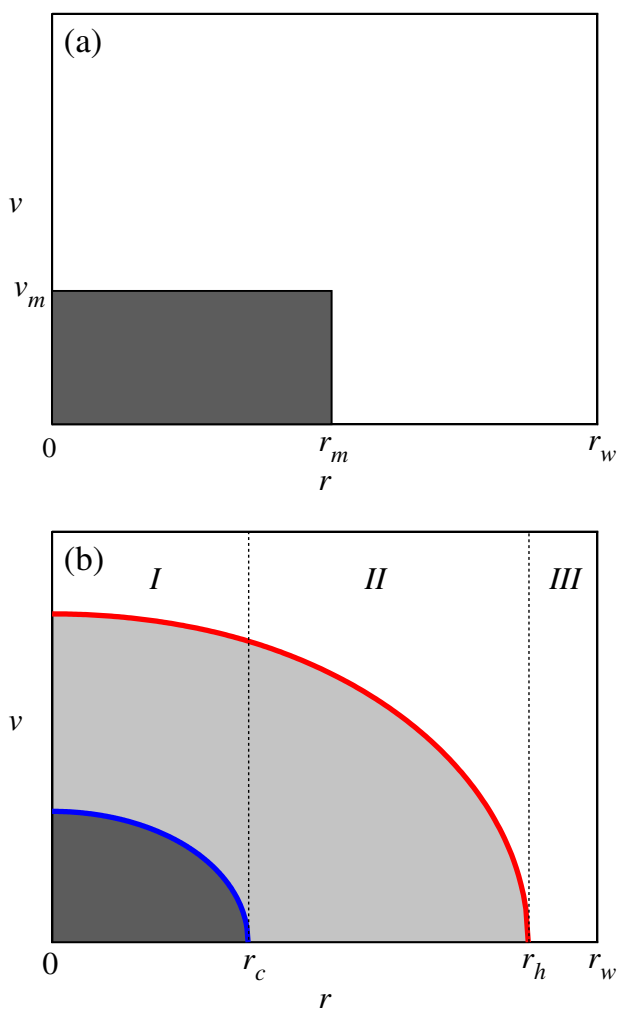

FIG. 1. Beam distributions in phase space. In (a), the gray area indicates the occupied regions for the initial waterbag distribution of Eq. (9) with maximum values for velocity $v_{m}$ and radius $r_{m}$. The density in phase space is uniform and equal to $N / \pi^{2} r_{m}^{2} v_{m}^{2}$ inside the distribution. Part (b) shows the proposed final stationary distribution formed by a dense core (dark gray) and a tenuous halo (light gray). Because of the characteristics of the Vlasov dynamics, the core density is assumed to be the same as the initial waterbag distribution, whereas the halo has a small fraction $\chi$ of the core density. The blue and red lines limit the core and the halo distributions and correspond to $\varepsilon(\mathbf{r}, \mathbf{v})=\varepsilon_{F}$ and $\varepsilon(\mathbf{r}, \mathbf{v})=\varepsilon_{h}$, respectively, where $\varepsilon(\mathbf{r}, \mathbf{v})$ is the single particle energy given by Eq. (3). For future reference, regions $I, I I$, and $I I I$ are presented and correspond, respectively, to $r<r_{c}, r_{c}<$ $r<r_{h}$, and $r_{h}<r<r_{w}$. 
the particle-core model resonance [9], for finite emittance beams it was observed that they typically spread uniformly in phase space up to the energy $\varepsilon_{h}$ [26,30]. Putting all this information together, we write the final stationary distribution as

$$
f_{s}(\mathbf{r}, \mathbf{v})=\frac{N}{\pi^{2} \epsilon_{0}^{2}}\left[\Theta\left(\varepsilon_{F}-\varepsilon\right)+\chi \Theta\left(\varepsilon_{h}-\varepsilon\right) \Theta\left(\varepsilon-\varepsilon_{F}\right)\right],
$$

where $\chi$ is the ratio between halo and core density in phase space. The distribution is represented in Fig. 1(b). Note that $f_{s}$ only depends on the phase-space coordinates through the single particle energy $\varepsilon$, defined in Eq. (3) and is consequently an equilibrium distribution. It depends on two still unknown parameters, $\varepsilon_{F}$ and $\chi$. These parameters will be determined self-consistently by imposing conservation of the number of particles and energy in the beam transport.

\section{B. Solving Poisson equation and conservation of the number of particles}

For the sake of calculations, we divide the space in three regions, $I, I I$, and $I I I$, that correspond, respectively, to $r<$ $r_{c}, r_{c}<r<r_{h}$, and $r_{h}<r<r_{w}$ [see Fig. 1(b)]. Here, $r_{c}$ is the maximum radius attained by the core particles. Integrating $f(\mathbf{r}, \mathbf{v})$ over the velocity space, we readily obtain the particle density in the three different regions, namely,

$$
\begin{gathered}
n_{I}(r)=\frac{2 N}{\pi \epsilon_{0}^{2}}\left[\varepsilon_{F}+\chi\left(\varepsilon_{h}-\varepsilon_{F}\right)-V_{I}(r)\right], \\
n_{I I}(r)=\frac{2 N \chi}{\pi \epsilon_{0}^{2}}\left[\varepsilon_{h}-V_{I I}(r)\right],
\end{gathered}
$$

and $n_{I I I}(r)=0, \quad$ where $V_{i}(r) \equiv \psi_{i}(r)+k_{0}^{2} r^{2} / 2, \quad i=$ $I, I I, I I I$ is the total potential that takes into account both self-field and external focusing contributions, and $r_{c}$ and $r_{h}$ can be determined by $V\left(r_{c}\right)=\varepsilon_{F}$ and $V\left(r_{h}\right)=\varepsilon_{h}$. Because of the continuity conditions on the self-fields, both $\psi_{i}(r)$ and $V_{i}(r)$ and their first derivatives have to be continuous at $r=r_{c}$ and $r=r_{h}$.

Substituting $n_{i}(r)$ in the Poisson equation (2) we obtain a closed set of equations for the self-field potential in the different regions. Let us start with region III which is free of charges and the potential has a general solution of the form $\psi_{I I I}(r)=D \log r+E$. The constants $D$ and $E$ can be easily determined by imposing the boundary condition at the wall, $\psi\left(r_{w}\right)=0$, and by noting that for any point located outside of the beam distribution, the potential is the same as if all the particles were concentrated in a line of charges located at $r=0$, leading to

$$
\psi_{I I I}(r)=-K \log \left(r / r_{w}\right)
$$

It is worth noting that Eq. (14) is already consistent with a normalized stationary distribution that satisfies $\int f_{s}(\mathbf{r}, \mathbf{v}) d \mathbf{r} d \mathbf{v}=N$.
Substituting $n_{I I}$, Eq. (13), and $\psi_{I I}=V_{I I}(r)-k_{0}^{2} r^{2} / 2$ in the Poisson equation, we then obtain an inhomogeneous modified Bessel equation for the total potential in region $I I$, whose general solution is

$$
V_{I I}(r)=B I_{0}\left(\alpha_{h} r\right)+C K_{0}\left(\alpha_{h} r\right)+\Delta,
$$

where $I_{m}$ and $K_{m}$ are the modified Bessel functions of $m$ th order, $\alpha_{h}=2(\chi K)^{1 / 2} / \epsilon_{0}$, and

$$
\Delta=\frac{k_{0}^{2}}{2 \alpha_{h}^{2}}\left(\alpha_{h}^{2} r_{h}^{2}-4\right)+K \log \left(\frac{r_{w}}{r_{h}}\right) .
$$

The constants $B$ and $C$ can be determined by the continuity of the total potential and its first derivative at $r=r_{h}$ leading to

$$
\begin{gathered}
B=\frac{2 k_{0}^{2} r_{h}}{\alpha_{h}} K_{1}\left(\alpha_{h} r_{h}\right)-\left(K-k_{0}^{2} r_{h}^{2}\right) K_{0}\left(\alpha_{h} r_{h}\right), \\
C=\frac{2 k_{0}^{2} r_{h}}{\alpha_{h}} I_{1}\left(\alpha_{h} r_{h}\right)+\left(K-k_{0}^{2} r_{h}^{2}\right) I_{0}\left(\alpha_{h} r_{h}\right) .
\end{gathered}
$$

Substituting $n_{I}$, Eq. (12), and $\psi_{I}=V_{I}(r)-k_{0}^{2} r^{2} / 2$ in the Poisson equation, we again obtain an inhomogeneous modified Bessel equation for the total potential, whose general solution can be cast in the form

$$
V_{I}(r)=A I_{0}\left(\alpha_{c} r\right)+\frac{A}{\chi}(1-\chi) I_{0}\left(\alpha_{c} r_{c}\right)+\Delta,
$$

where $\alpha_{c}=2 K^{1 / 2} / \epsilon_{0}, \Delta$ is given in Eq. (16), and we have already used $V\left(r_{c}\right)=\varepsilon_{F}$ in order to explicitly eliminate $\varepsilon_{F}$ from the expression. By imposing the continuity of the first derivative of the total potential at $r=r_{c}$, we can express the constant $A$ as

$$
A=\chi^{1 / 2} \frac{B I_{1}\left(\alpha_{h} r_{c}\right)-C K_{1}\left(\alpha_{h} r_{c}\right)}{I_{1}\left(\alpha_{c} r_{c}\right)} .
$$

In order to complete the determination of the potential we still need to impose its continuity at $r=r_{c}$, i.e.,

$$
V_{I}\left(r_{c}\right)=V_{I I}\left(r_{c}\right)
$$

Equation (21) is a transcendental equation that along with the conservation of energy, to be derived in the following subsection, will be used to determine the two unknown parameters $r_{c}$ and $\chi$ of the model. The equation guarantees that the self-field potential of the stationary distribution is consistent with the beam normalization condition $\int f_{s}(\mathbf{r}, \mathbf{v}) d \mathbf{r} d \mathbf{v}=N$.

\section{Conservation of energy}

In order to calculate the average energy per particle of the stationary beam, we start by determining its envelope squared $r_{b s}^{2}=2\left\langle r^{2}\right\rangle=2 \int r^{2} n(r) r d r$. Using Eqs. (12), (13), (15), and (19), we obtain 


$$
\begin{aligned}
r_{b s}^{2}= & {\left[2+\frac{k_{0}^{2}\left(8-\alpha_{h}^{2} r_{h}^{2}\right)}{\alpha_{h}^{2} K}\right] r_{h}^{2}-\frac{8 A r_{c}^{2}}{K}\left[\frac{(1-\chi) I_{0}\left(\alpha_{c} r_{c}\right)}{2 \chi}+\frac{I_{1}\left(\alpha_{c} r_{c}\right)}{\alpha_{c} r_{c}}\right] } \\
& +\frac{8 r_{c}\left[B I_{1}\left(\alpha_{h} r_{c}\right)-C K_{1}\left(\alpha_{h} r_{c}\right)\right]-8 r_{h}\left[B I_{1}\left(\alpha_{h} r_{h}\right)-C K_{1}\left(\alpha_{h} r_{h}\right)\right]}{\alpha_{h} K} .
\end{aligned}
$$

To determine the beam self-field energy for the stationary distribution, we need to perform the integral in Eq. (8). Although this integral can be calculated analytically, in order to simplify the result we can perform an approximation. Namely, because the halo distribution is tenuous, we can disregard the detailed distribution of halo particles in the region $r_{c}<r<r_{h}$ and assume, for instance, that they are all at $r=r_{c}$. With that, the whole region $r_{c}<r<r_{w}$ is free of charge with a self-field potential given by Eq. (14). The self-field energy of the stationary state is then given by

$$
\begin{aligned}
\mathcal{E}_{\psi s}= & \frac{r_{c}^{2}}{8 K}\left\{k_{0}^{4} r_{c}^{2}+2 A^{2} \alpha_{c}^{2} I_{1}^{2}\left(\alpha_{c} r_{c}\right)-2 A\left[4 k_{0}^{2}\right.\right. \\
& \left.\left.+A \alpha_{c}^{2} I_{0}\left(\alpha_{c} r_{c}\right)\right] I_{2}\left(\alpha_{c} r_{c}\right)\right\}-K \log \left(\frac{r_{c}}{r_{w}}\right),
\end{aligned}
$$

where use has been made of $\psi_{I}=V_{I}(r)-k_{0}^{2} r^{2} / 2$ and Eq. (19) for $r<r_{c}$. Expression (23) has been tested against the exact value of the self-energy and for the cases analyzed here represented a variation of the order of $1 \%$ or less in the total energy of the beam (see caption of Fig. 3). Anyway, for largely mismatched beams as well as very space-charge dominated beams, one should consider using the exact self-energy expression to guarantee accuracy.

To complete the determination of the average energy per particle given by Eq. (7), we also need to compute $\left\langle v^{2}\right\rangle$ for the stationary distribution. However, if we notice that $f_{s}$ is an equilibrium distribution then its envelope must correspond to a matched envelope. Substituting Eq. (5) in (6), we can conveniently write the matching condition as

$$
2\left\langle v^{2}\right\rangle=k_{0}^{2} r_{b}^{2}-K,
$$

valid for any equilibrium distribution. Note that the term $\left\langle r v_{r}\right\rangle$ in Eq. (5) is proportional to the derivative of the envelope with respect to $s$ and therefore vanishes for a stationary beam. Using this in the expression for the average energy per particle for the stationary distribution and imposing conservation of energy leads to

$$
\frac{k_{0}^{2} r_{b s}^{2}}{2}-\frac{K}{4}+\mathcal{E}_{\psi s}=\mathcal{E}_{0}
$$

where $r_{b s}, \mathcal{E}_{\psi s}$, and $\mathcal{E}_{0}$ are given, respectively, by Eqs. (22), (23), and (10). Equation (25) is another transcendental equation that has to be solved numerically. Solving Eqs. (21) and (25) for the unknown parameters $r_{c}$ and $\chi$ completely determines the final stationary state.

\section{Emittance growth and halo fraction}

Once the final stationary state has been determined, we can compute the total emittance growth that occurs in the beam relaxation process. Substituting Eq. (24) in Eq. (5), we obtain for the final stationary emittance

$$
\epsilon_{s}=r_{b s} \sqrt{k_{0}^{2} r_{b s}^{2}-K},
$$

where $r_{b s}$ is given in Eq. (22). The emittance growth is given by $\epsilon_{s} / \epsilon_{0}$.

Regarding the halo formation, an important quantity is the fraction of particles that evaporate to the halo during the relaxation process. Taking into account the stationary distribution in Eq. (11), this quantity corresponds to the fraction of particles with energies between $\epsilon_{F}$ and $\epsilon_{h}$ [see Fig. 1(b)], i.e., $\mathcal{F}_{h}=\left(\chi / \pi^{2} \epsilon_{0}^{2}\right) \int \Theta\left(\varepsilon_{h}-\varepsilon\right) \Theta(\varepsilon-$ $\left.\varepsilon_{F}\right) d \mathbf{r} d \mathbf{v}$. Performing the integral we find

$$
\mathcal{F}_{h}=1-\frac{2 A r_{c}^{2} I_{2}\left(\alpha_{c} r_{c}\right)}{\epsilon_{0}^{2}}
$$

\section{E. Beams with different initial distributions}

So far, we have only considered the relaxation of beams with an initial distribution given by the waterbag distribution, in Eq. (9). In general, however, we may expect initial distributions that present a nonuniform density in phase space. In order to handle such cases, we can discretize the nonuniform distribution into $p$ levels [30]. While this procedure allows for a very detailed description of the beam, it demands an equally detailed knowledge of the beam initial distribution. In many practical situations, however, there is no such knowledge and all that is known from the initial beam are the rms quantities, like the envelope and the emittance. Taking this into consideration, we take the lowest order $p=1$ and approximate any given initial distribution by Eq. (9) with the envelope $r_{m}$ and emittance $\epsilon_{0}$ corresponding to the actual beam. With this, we can estimate the final stationary state, the emittance growth, and the halo fraction for any beam, just based on its initial envelope and emittance. Self-consistent simulations are presented in the next section to verify the validity of this approximation.

\section{NUMERICAL RESULTS}

In order to test the theory presented, we perform $N$-particle self-consistent simulations. The simulations are based on Gauss's law where the field at a certain radial coordinate $r$ depends on the total number of particles with 
coordinates smaller than $r$ [4]. This method precludes the effects of collisions between individual particles and is convenient because instabilities and profile distortions around the round shape are not expected here [33,34]. In the simulations we launch $N=5000$ macroparticles according to a prescribed distribution and evolve them through long axial distances of the order of $10^{3}-10^{4}$ betatron oscillations. When the emittance of the beam reaches a plateau, we consider that the beam has reached its stationary state. We consider three different initial beam distributions, namely, a waterbag given by Eq. (9), a semi-Gaussian distribution, and a full Gaussian distribution both in space and velocity. The analysis is simplified if we measure longitudinal and transverse coordinates in units of $k_{0}^{-1}$ and $\left(\epsilon_{0} / k_{0}\right)^{1 / 2}$, respectively. Then, the initial beam is characterized by two parameters only: $K / k_{0} \epsilon_{0}$ and the mismatch parameter $\mu$. In the results presented below, the halo size used in the theory is not directly obtained by the particle-core model, but rather, the one approximated by the empirical formula proposed by Ref. [5], namely, $r_{h}=2 r_{b}^{*}(0)(1+\log \mu)$, where $r_{b}^{*}(0)$ is the initial matched beam envelope obtained from Eq. (6) with $\varepsilon=\varepsilon_{0}$.

In Fig. 2, we compare the final stationary particle distribution obtained from the theory (solid lines) and the $N$-particle simulation (dots) for three different cases. In panel (a) we present the results for an initial waterbag distribution with $K / k_{0} \epsilon_{0}=0.1$ and $\mu=1.5$. This parameter set corresponds to a mildly space-charge dominated beam that is comparable to that found in the experiments of Ref. [7]. Despite the small space-charge forces a large halo is apparent. Clearly, the model agrees very well with the simulation results, describing very closely both the core and the halo particle distributions. In panel (b), we consider a beam with the same initial distribution as in (a), but with larger space-charge forces corresponding to $K / k_{0} \epsilon_{0}=1.0$. Again, a very good agreement is found. In panel (c), we present an example with a different initial distribution. In particular, we consider the same parameters as in panel (b), namely $K / k_{0} \epsilon_{0}=1.0$ and $\mu=1.5$, but now for a fully Gaussian distribution. As expected, because the initial distribution is nonuniform both in the configuration and the velocity space, the final agreement between the final stationary state reached in the simulation and the theory is not as impressive as in the previous cases. Nevertheless, taking into consideration the crudeness and simplicity of the model, the results are still quite satisfactory, particularly concerning the halo distribution which is reasonably close to the actual one. For the sake of comparison, we also present in Fig. 2 the respective initial normalized charge distributions (dashed curves).

Next, we compare the emittance growth calculated from the model and obtained from the simulations with different initial conditions. These results are presented in Fig. 3 as a function of the mismatch parameter for $K / k_{0} \epsilon_{0}=0.1$ (a) and $K / k_{0} \epsilon_{0}=1.0(\mathrm{~b})$. The theoretical results are found to be in good agreement with the numerical results. The agreement is better for the less space-charge dominated case. As the space charge is increased, the emittance seems to become more sensitive to the details of the stationary distribution, requiring a more involved description of the stationary state if a more accurate prediction of the emittance growth is required [30]. Nevertheless, as seen in
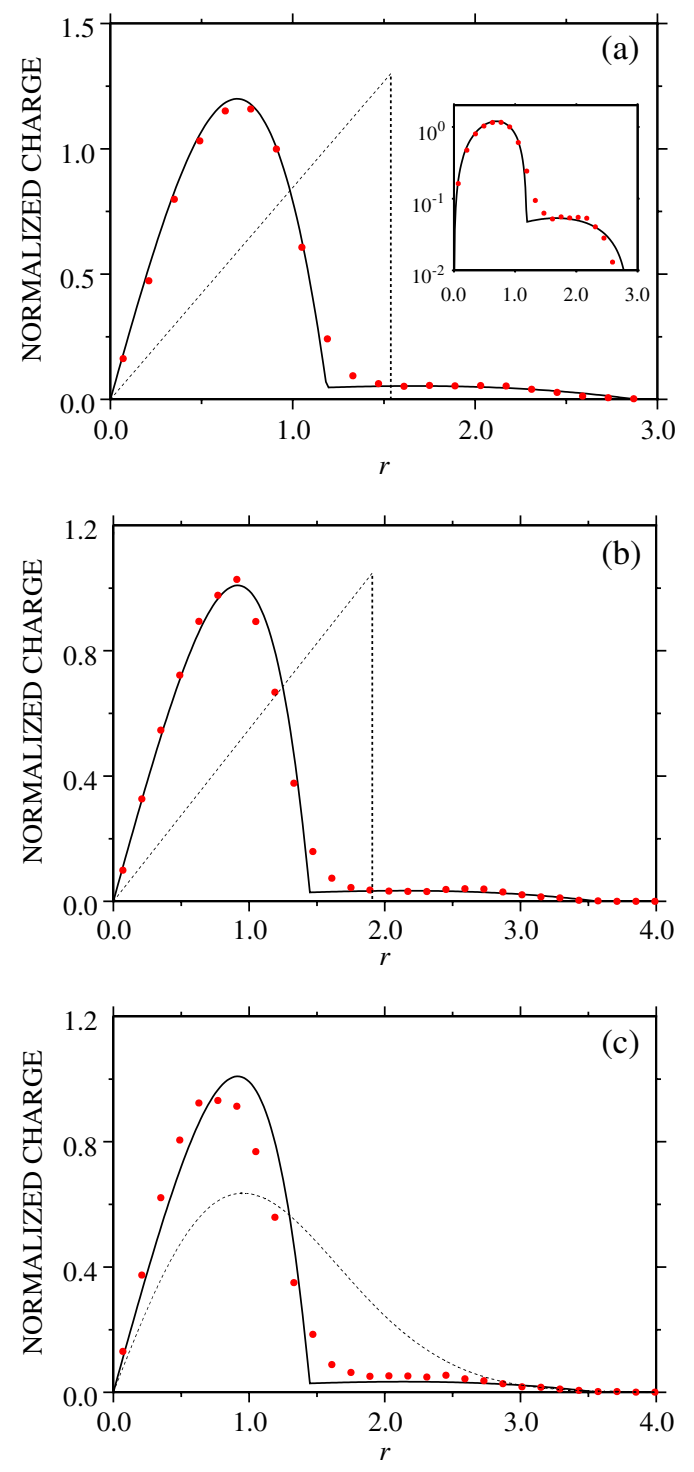

FIG. 2. Comparison of the normalized charge as a function of radius obtained from the theory (solid curve) and the $N$-particle simulation (red dots). The space-charge parameter and the initial distributions considered are: $K / k_{0} \epsilon_{0}=0.1$ and waterbag in (a), $K / k_{0} \epsilon_{0}=1.0$ and waterbag in (b), and $K / k_{0} \epsilon_{0}=1.0$ and fully Gaussian in (c). In all the cases, $\mu=1.5, r$ is measured in units of $\left(\epsilon_{0} / k_{0}\right)^{1 / 2}$, and the charge is normalized to the total charge $N$ in the beam. For the sake of comparison, we also present the respective initial normalized charge distributions (dashed curves). The inset in (a) presents the normalized charge in log form, showing that despite the large difference in core and halo density, the theory agrees well with the simulation. 

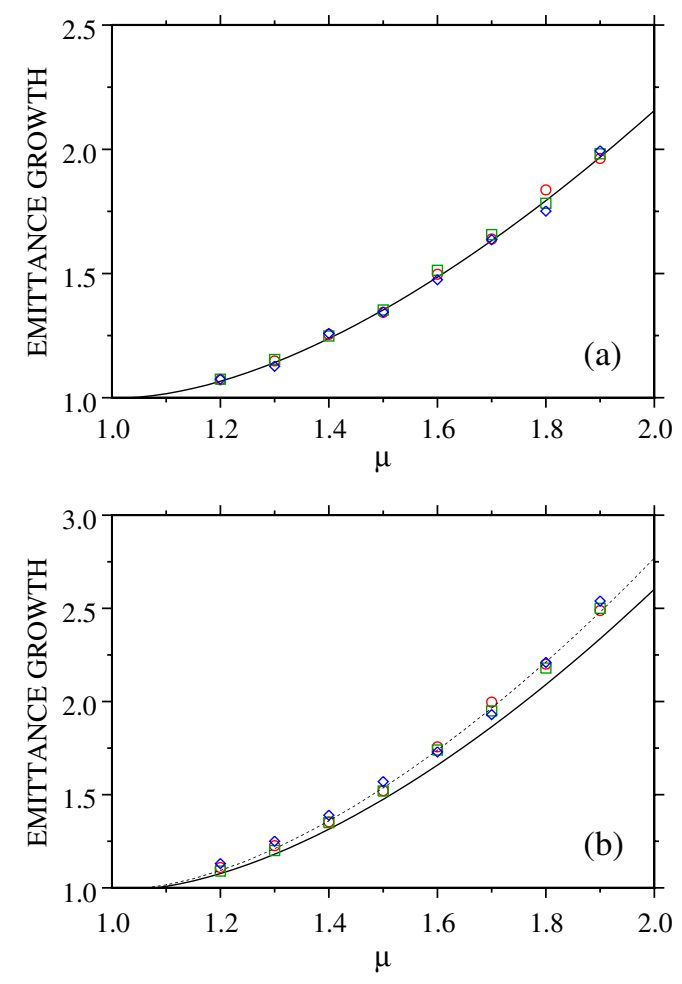

FIG. 3. Emittance growth $\epsilon_{s} / \epsilon_{0}$ as a function of the mismatch parameter $\mu$ obtained from the theory (solid curve) and the $N$-particle simulations (symbols) for $K / k_{0} \epsilon_{0}=0.1$ in (a) and $K / k_{0} \epsilon_{0}=1.0$ in (b). The symbols correspond to the different initial distribution in the simulation: waterbag (circle), semiGaussian (square), and Gaussian beam (diamond). The dashed line in panel (b) corresponds to the emittance obtained using the exact form of the self-energy instead of the approximation given by Eq. (23). It shows that an improved estimate can be obtained by using the exact self-energy expression for larger mismatches and more space-charge dominated beams.

Fig. 3, the simplified model presented here already provides a good estimate of the emittance growth. Another interesting feature seen in Fig. 3 is that the emittance growth is rather independent of the initial beam distribution. This may be credited to the fact that the emittance value is more affected by the halo particles than the core distribution. And, as indicated in Fig. 2, changes in the initial distribution of mismatched beams seem to affect more the stationary core distribution than the halo.

We next apply the theory to estimate the fraction of particles that evaporate from the core to form the halo. In Fig. 4, we show $\mathcal{F}_{h}$ as a function of the mismatch parameter $\mu$ obtained from the theory, Eq. (27), for $K / k_{0} \epsilon_{0}=0.1$ (solid line) and $K / k_{0} \epsilon_{0}=1.0$ (dashed line). A nearly linear dependence of the halo fraction with the mismatch parameter is observed. The figure also indicates that the halo fraction decreases as the space charge is increased. In fact, this trend is verified by computing $\mathcal{F}_{h}$ as a function of $K / k_{0} \epsilon_{0}$ for fixed $\mu$ (not shown). We note that, although the halo fraction is a decreasing function of $K / k_{0} \epsilon_{0}$, the total

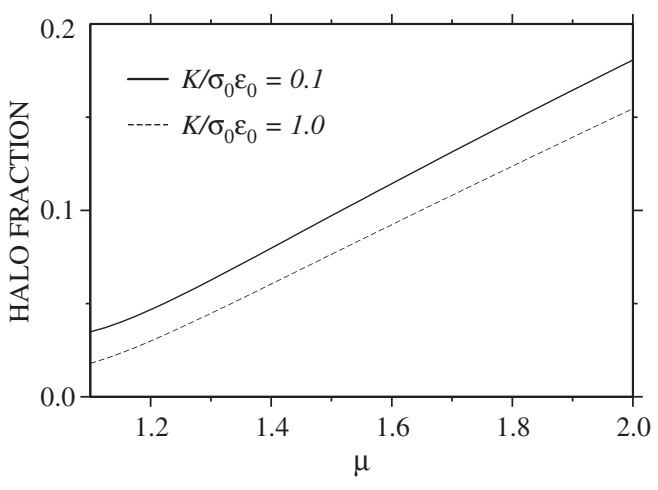

FIG. 4. Halo fraction $\mathcal{F}_{h}$ as a function of the mismatch parameter $\mu$ obtained from the theory, Eq. (27), for $K / k_{0} \epsilon_{0}=0.1$ (solid line) and $K / k_{0} \epsilon_{0}=1.0$ (dashed line).

charge in the halo, given by $K \mathcal{F}_{h}$, grows as the beam becomes more intense. The halo fraction was not compared to numerical results from the simulations because there is no satisfactory criteria to separate halo and core particles in the simulation.

As far as emittance growth is concerned, the results presented in Fig. 3(a) for a low space-charge beam are very similar to those obtained from the free-energy model described in Ref. [1]. Thus, our emittance growth estimates should also agree very well with the experimental results presented in Ref. [7]. Nevertheless, in contrast to the freeenergy model [1], the theory derived here not only allows for emittance growth estimates, but also provides a good description of the final stationary distribution attained by the beam, including halo density and fraction. Therefore, it would be interesting to validate the model against experimental results of halo formation in mismatched spacecharge dominated beams [7,35-37].

\section{CONCLUSION}

A simplified theoretical model that allows one to predict the final stationary state attained by an initially mismatched beam is presented. The proposed stationary state is described by a core-halo distribution. Based on the incompressibility of the Vlasov dynamics which governs the beam evolution, the core is assumed to be a fully degenerate Fermi-Dirac distribution, where the particles occupy the lowest possible energy states accessible to them. The halo, on the other hand, is given by a tenuous uniform distribution that extends up to a maximum energy determined by the core-particle resonance. This leads to a self-consistent model for which the beam density and selffields can be determined analytically as a function of two parameters-the core size and the halo density. This parameters are in turn determined by numerically solving two algebraic equations that correspond to the conservation of the total number of particles and the energy of the system. The theory allows one to estimate important quantities, such as the emittance growth and the fraction of particles 
that evaporate to the halo in the relaxation process. In particular, regarding the halo fraction, the model foresees a nearly linear increase with the mismatch amplitude, as well as an inverse dependence with the space-charge parameter $K / k_{0} \epsilon_{0}$. Self-consistent $N$-particle simulations were performed to verify the predictions of the theory. The simulations show that the emittance growth is rather independent of the details of the initial distribution, being well characterized by the initial rms quantities of the beam, namely, the envelope and initial emittance. Moreover, good agreement is found between the particle distributions predicted by the model and obtained in the simulations, particularly regarding the halo description. The predicted emittance growths are also found to agree with the simulations.

\section{ACKNOWLEDGMENTS}

This work was supported by CNPq and FAPERGS, Brazil, and by the U.S.-AFOSR under Grant No. FA9550-09-1-0283.

[1] M. Reiser, J. Appl. Phys. 70, 1919 (1991).

[2] M. Reiser, Theory and Design of Charged Particle Beams (Wiley-InterScience, New York, 1994).

[3] R. L. Gluckstern, Phys. Rev. Lett. 73, 1247 (1994).

[4] H. Okamoto and M. Ikegami, Phys. Rev. E 55, 4694 (1997).

[5] T.P. Wangler, K. R. Crandall, R. Ryne, and T. S. Wang, Phys. Rev. ST Accel. Beams 1, 084201 (1998).

[6] C. Chen and R. Pakter, Phys. Plasmas 7, 2203 (2000); R. Pakter and C. Chen, IEEE Trans. Plasma Sci. 28, 502 (2000).

[7] C. K. Allen, K. C. D. Chan, P. L. Colestock, K. R. Crandall, R. W. Garnett, J. D. Gilpatrick, W. Lysenko, J. Qiang, J. D. Schneider, M. E. Schulze, R. L. Sheffield, H. V. Smith, and T. P. Wangler, Phys. Rev. Lett. 89, 214802 (2002).

[8] K. Fiuza, F. B. Rizzato, and R. Pakter, Phys. Plasmas 13, 023101 (2006).

[9] R.P. Nunes, R. Pakter, and F. B. Rizzato, Phys. Plasmas 14, 023104 (2007); J. Appl. Phys. 104, 013302 (2008).

[10] M. Hess and C. Chen, Phys. Plasmas 7, 5206 (2000); Phys. Lett. A 295, 305 (2002); Phys. Rev. ST Accel. Beams 7, 092002 (2004).

[11] J. S. Moraes, R. Pakter, and F. B. Rizzato, Phys. Rev. Lett. 93, 244801 (2004); Phys. Plasmas 12, 023104 (2005).

[12] M. Hess, IEEE Trans. Plasma Sci. 36, 729 (2008).

[13] L. C. Martins, F. B. Rizzato, and R. Pakter, J. Appl. Phys. 106, 043305 (2009).
[14] O. A. Anderson, Part. Accel. 21, 197 (1987).

[15] S. Bernal, R. A. Kishek, M. Reiser, and I. Haber, Phys. Rev. Lett. 82, 4002 (1999).

[16] S. G. Anderson and J. B. Rosenzweig, Phys. Rev. ST Accel. Beams 3, 094201 (2000).

[17] S. M. Lund, D.P. Grote, and R.C. Davidson, Nucl. Instrum. Methods Phys. Res., Sect. A 544, 472 (2005).

[18] F. B. Rizzato, R. Pakter, and Y. Levin, Phys. Plasmas 14, 110701 (2007).

[19] R. P. Nunes, R. Pakter, F. B. Rizzato, A. Endler, and E. G. Souza, Phys. Plasmas 16, 033107 (2009).

[20] E. G. Souza, A. Endler, R. Pakter, F. B. Rizzato, and R. P. Nunes, Appl. Phys. Lett. 96, 141503 (2010).

[21] B. L. Qian, J. Zhou, and C. Chen, Phys. Rev. ST Accel. Beams 6, 014201 (2003).

[22] J. Zhou, B. L. Qian, and C. Chen, Phys. Plasmas 10, 4203 (2003).

[23] R. Pakter, Y. Levin, and F. B. Rizzato, Appl. Phys. Lett. 91, 251503 (2007).

[24] S. M. Lund, J. J. Barnard, and J. M. Miller, in Proceedings of the Particle Accelerator Conference, Dallas, TX, 1995 (IEEE, New York, 1995), p. 3278.

[25] E. A. Startsev and S. M. Lund, Phys. Plasmas 15, 043101 (2008).

[26] Y. Levin, R. Pakter, and T. N. Teles, Phys. Rev. Lett. 100, 040604 (2008).

[27] Y. Levin, R. Pakter, and F. B. Rizzato, Phys. Rev. E 78, 021130 (2008).

[28] F. B. Rizzato, R. Pakter, and Y. Levin, Phys. Rev. E 80, 021109 (2009).

[29] T. N. Teles, Y. Levin, R. Pakter, and F. B. Rizzato, J. Stat. Mech. (2010) P05007.

[30] T. N. Teles, R. Pakter, and Y. Levin, Appl. Phys. Lett. 95, 173501 (2009).

[31] R. C. Davidson and H. Qin, Physics of Intense Charged Particle Beams in High Energy Accelerators (World Scientific, Singapore, 2001).

[32] The distribution in Eq. (9) is not to be confused with a waterbag distribution in energy which may represent an equilibrium beam distribution, as discussed in Ref. [31], pp. 217-219.

[33] I. Hofmann, L. J. Laslett, L. Smith, and I. Haber, Part. Accel. 13, 145 (1983).

[34] W. Simeoni Jr., F. B. Rizzato, and R. Pakter, Phys. Plasmas 13, 063104 (2006).

[35] E.P. Gilson, R.C. Davidson, P.C. Efthimion, and R. Majeski, Phys. Rev. Lett. 92, 155002 (2004).

[36] R. Takai, H. Enokizono, K. Ito, Y. Mizuno, K. Okabe, and H. Okamoto, Jpn. J. Appl. Phys. 45, 5332 (2006).

[37] H. Higaki, S. Fujimoto, K. Fukata, K. Ito, M. Kuriki, H. Okamoto, and J. Aoki, in Proceedings of the 2010 International Accelerator Conference (to be published). 